\title{
Crystal-proven Gout and Characteristic Gout Severity Factors Are Associated with Cardiovascular Disease
}

\author{
Iris J.M. Disveld, Jaap Fransen, Gerard A. Rongen, Laura B.E. Kienhorst, Sahel Zoakman, \\ Hein J.E.M. Janssens, and Matthijs Janssen
}

\begin{abstract}
Objective. Our aim was to examine the prevalence of cardiovascular disease (CVD) in patients with crystal-proven gout compared to arthritis controls. Further, we analyzed the association between characteristic gout severity factors and CVD to provide further support for a pathogenetic relationship between gout and CVD.

Methods. Patients with arthritis referred for diagnosis were consecutively included in the Gout Arnhem-Liemers cohort. Joint fluid analysis was performed in all referred patients; controls were negative for crystals. Patients' characteristics and different manifestations of CVD and gout severity factors (disease duration, attack frequency, tophi, affected joints, high serum urate acid level, joint damage) were collected. Gout patients were compared with controls for the prevalence of CVD. In addition, the association between characteristic gout severity factors and presence of CVD was analyzed.

Results. Data from 700 gout patients and 276 controls were collected. CVD was present in 47\% (95\% CI $44 \%-51 \%$ ) and $24 \%$ (95\% CI 19\%-29\%) of gout patients and controls, respectively. Corrected for confounders, gout was still strongly associated with an increased prevalence of CVD compared to controls (OR 3.39, 95\% CI 2.37-4.84). In patients with gout, disease duration $\geq 2$ years, oligo- or polyarthritis, serum urate acid $>0.55 \mathrm{mmol} / \mathrm{l}$ at presentation, and joint damage were independently ( $\mathrm{p}<0.05$ ) associated with prevalent CVD.

Conclusion. Crystal-proven gout was strongly associated with an increased prevalence of CVD. In patients with gout, characteristic gout severity factors were associated with CVD. (First Release April 15 2018; J Rheumatol 2018;45:858-63; doi:10.3899/jrheum.170555)
\end{abstract}

Key Indexing Terms: GOUTY ARTHRITIS

CARDIOVASCULAR DISEASES

OUTCOMES

Gout is a disease affecting many patients worldwide ${ }^{1}$. One of the key metabolic features of gout is the supersaturation of urate in the serum, called hyperuricemia ${ }^{2}$. Hyperuricemia can result in multiple depositions of monosodium urate (MSU) crystals, including intraarticular deposition, resulting in bouts of acute arthritis leading to acutely painful joint

From the Department of Rheumatology, Rijnstate Hospital, Arnhem; Department of Rheumatology, Radboud University Medical Centre; Departments of Internal Medicine, and Pharmacology and Toxicology, Radboud University Medical Centre; Department of Primary and Community Care, Radboud University Medical Centre, Nijmegen; Department of Dermatology, University Medical Centre Utrecht, Utrecht, the Netherlands.

I.J. Disveld, MD, Department of Rheumatology, Rijnstate Hospital; J. Fransen, PhD, Department of Rheumatology, Radboud University Medical Centre; G.A. Rongen, Professor, Department of Internal Medicine, and Department of Pharmacology and Toxicology, Radboud University Medical Centre; L.B. Kienhorst, MD, LLM, Department of Dermatology, University Medical Centre Utrecht; S. Zoakman, MD, Department of Rheumatology, Rijnstate Hospital; H.J. Janssens, MD, PhD, Department of Primary and Community Care, Radboud University Medical Centre; M. Janssen, MD, PhD, Department of Rheumatology, Rijnstate Hospital.

Address correspondence to Dr. M. Janssen, Department of Rheumatology, Rijnstate Hospital, PO Box 9555, 6800 TA Arnhem, the Netherlands. E-mail: matthijsjanssen@icloud.com

Accepted for publication December 12, 2017. inflammation manifesting as acute gout. Interestingly, other sites of urate deposition may remain asymptomatic.

After crystals form, they constantly accumulate as long as hyperuricemia persists. If the crystals are dissolved through treatment, the gout is cured ${ }^{2,3}$. This longterm condition produces tophi, which are solid depositions of MSU crystals formed in joints and soft tissues. Ultimately, crystal-mediated inflammation and intraarticular tophi may cause erosion of the cartilage and bone, resulting in joint damage ${ }^{4}$.

In addition to its disease manifestations in joints, gout appears to be associated with an increased risk of cardiovascular disease (CVD) $)^{2,5,6,7,8}$. When an association of gout and increased risk of CVD is proven, then a right and timely diagnosis and treatment will become more mandatory. Several studies showed a high prevalence of CVD in patients with gout, raising the question of whether gout is a risk factor for $\mathrm{CVD}^{9,10,11,12}$. However, these studies were based on the clinical diagnosis of gout, although the gold standard for diagnosing gout is the identification of MSU crystals in joint fluid by polarization microscopy ${ }^{13}$. Therefore, previous reports could have misdiagnosed as gout other diseases that cause arthritis, which might have introduced bias. We could not identify studies associating crystal-proven gout and the

Personal non-commercial use only. The Journal of Rheumatology Copyright @ 2018 . All rights reserved. 
prevalence of CVD in secondary care populations. In addition, none of the previous studies had addressed the question of whether the prevalence of CVD in patients with gout is related with gout disease severity factors or features of chronic gout, with the exception of studies investigating the association of hyperuricemia with $\mathrm{CVD}^{2,14}$.

Because CVD is the leading global cause of death, it is interesting to gain insight into the association between crystal-proven gout and CVD, to understand the extent of the problem. If there indeed is an association between gout and CVD, and when disease severity in gout is associated with CVD, there might be a pathophysiological link between gout and the development of CVD.

Therefore, the objectives were to analyze the following: (1) what is the prevalence of CVD in patients with crystal-proven gout at the moment of gout diagnosis, compared with non-gout arthritis controls; and (2) are gout severity factors related to the prevalence of CVD in patients with crystal-proven gout, at the moment of gout diagnosis?

\section{MATERIALS AND METHODS}

Design. This was a cross-sectional study of patients with arthritis referred to the rheumatologist, by primary care or secondary care physicians, for diagnosis in our regional gout research center (Rijnstate Hospital, Arnhem, the Netherlands). Patients with signs and symptoms of arthritis were consecutively included in the Gout Arnhem-Liemers (GOAL) cohort, and data were stored in a database of the rheumatology department. Our study comprised patients with gout and non-gout acute monoarthritis consecutively included from July 2011 until May 2016.

First, patients with crystal-proven gout were compared with non-gout monoarthritis controls for the prevalence of CVD, while correcting for confounders. Second, an analysis was performed in patients with crystal-proven gout to determine whether gout disease severity factors were associated with CVD. At the first visit of our outpatient rheumatology clinic, patients gave written informed consent for the use of their clinical data and for blood sampling. At inclusion, patients had a standardized interview and physical examination. Ethical approval for this type of study was not required in accordance with the policy of the Rijnstate hospital.

Diagnosis. The affected joint of each patient was aspirated, and the joint fluid was analyzed with a polarization microscope for the presence of MSU crystals to diagnose patients as having either crystal-proven gout or other forms of arthritis. Patients with other forms of arthritis formed the control group, which included mainly patients with a reactive arthritis, bacterial arthritis, calcium pyrophosphate dihydrate (CPPD) crystal deposition disease, psoriatic arthritis (PsA), and unclassified monoarthritis. When no joint fluid could be collected during arthrocentesis, the patient was classified as having non-gout. For control patients with clinical features of gout without visualization of MSU crystals and for patients with unclassified monoarthritis, we used the Gout Diagnostic Decision Rule as a proxy for diagnosing gout ${ }^{15,16}$. If the Gout Diagnostic Decision Rule indicated a high probability of gout (score $>8$ ) we considered this arthritis as caused by gout but not crystal-proven, and these patients were excluded from entry in the study.

Data collection. The collected data at the point of diagnosis of either crystal-proven gout or other forms of arthritis included clinical characteristics, CV risk factors, and CVD. The patients' clinical characteristics and $\mathrm{CV}$ risk factors recorded in the database included age, sex, race, body mass index (BMI), family history of CVD, smoking, alcohol consumption, serum urate (SU) level, serum creatinine level, glomerular filtration rate (GFR) as estimated by renal creatinine clearance, diabetes mellitus, hypertension
(HTN), and hypercholesterolemia. CVD was defined as $\geq 1$ of the following 8 diseases: angina pectoris, myocardial infarction, heart failure, transient ischemic attack, cerebrovascular accident, peripheral vascular disease, heart valve abnormality, or arrhythmia. All clinical characteristics, CV risk factors, and CVD were reported if these were physician-diagnosed or reported in patient records and double-checked by asking patients for the presence of $\mathrm{CV}$ risk factors and CVD at the inclusion visit. BMI, serum creatinine level, and GFR (as estimated by renal creatinine clearance) were measured at the inclusion visit.

In addition, in patients with gout, gout disease severity factors were collected at the inclusion visit. In total, 6 gout disease severity factors were defined as the following: disease duration $\geq 2$ years, attack frequency $\geq 4$ attacks/year, tophaceous gout, oligo- or polyarthritis, high SU acid $(>0.55$ $\mathrm{mmol} / \mathrm{l}$ ), and presence of gout-related joint damage. Disease duration was defined at inclusion as the time between the first gout episode and diagnosis of crystal-proven gout. The first gout episode was recorded using patient records of family physicians. In addition, patients were asked for the date of the first gout episode and the attack frequency. The presence of tophi and number of affected joints (mono-, oligo-, or polyarthritis) were recorded after physical examination at the inclusion visit. Finally, at inclusion the SU acid level was measured, and radiographs were performed to detect gout-related joint damage at inclusion ${ }^{17,18}$.

Statistical analysis clinic. To analyze the prevalence of CVD in patients with crystal-proven gout compared to non-gout arthritis controls, we calculated the prevalence of CVD among patients with crystal-proven gout and non-gout monoarthritis controls. We performed univariate logistic regression analysis to quantify the association between crystal-proven gout and the presence of CVD, using the OR. Patients' characteristics were compared between patients with crystal-proven gout and control patients using univariate logistic regression analyses, and any potential confounder $(\mathrm{p}<0.05)$ was included in subsequent multivariate models. Confounder correction was attained by adding potential confounders 1 at a time in a multiple logistic regression model; a change of the main effect by $\geq 10 \%$ was the selection criterion.

To analyze whether disease severity was associated with CVD in gout, patients' characteristics and gout disease severity factors between patients with crystal-proven gout with and without CVD were compared using univariate logistic regression analyses, and OR were calculated.

All variables with a univariate $\mathrm{p}<0.05$ and $\mathrm{OR}>1$ were selected. Subsequently, full-model multiple logistic regression analyses were performed with backward selection and $\mathrm{p}<0.05$ as selection criterion.

For all measures, we calculated $95 \%$ CI. All reported $\mathrm{p}$ values are 2-sided. All statistical tests were performed using SPSS software version 20.0 (SPSS Inc.).

\section{RESULTS}

From July 2011 until May 2016, 700 patients with crystal-proven gout and 336 controls were consecutively included in the GOAL cohort. In the control group, 179 patients showed clinical features of gout without visualization of MSU crystals or were diagnosed as having unclassified monoarthritis. For 60 of the 179 controls, the Gout Diagnostic Decision Rule indicated a high probability of gout (score $>8$ ) and these control patients were therefore excluded from entry in our study.

In total, 700 patients with crystal-proven gout and 276 non-gout arthritis controls were enrolled in the crosssectional analysis. The control group consisted of reactive arthritis, bacterial arthritis, CPPD crystal deposition disease, and PsA, but most control patients with monoarthritis had a self-limiting form of unclassified monoarthritis. The clinical

Personal non-commercial use only. The Journal of Rheumatology Copyright @ 2018. All rights reserved. 
characteristics and $\mathrm{CV}$ risk factors of patients and controls, and the prevalence of CVD, are shown in Table 1. The mean (SD) levels of SU acid in gout and non-gout patients were comparable: 0.45 (0.13) and $0.43(0.13) \mathrm{mmol} / \mathrm{l}$ ( $\mathrm{p}$ was nonsignificant).

In total, $93 \%$ of the population was white. Data analysis revealed a statistically significant greater prevalence of CVD in patients with crystal-proven gout as compared with non-gout arthritis controls. Compared with controls, individuals with crystal-proven gout had a higher prevalence of angina pectoris, myocardial infarction, heart failure, transient ischemic attack, cerebrovascular accident, peripheral vascular disease, heart valve abnormality, and arrhythmia. These differences were statistically significant, except for transient ischemic attack (Table 1).

Crystal-proven gout was also associated with an increased prevalence of CVD compared to non-gout arthritis controls in univariate logistic regression analysis (Table 1 and Table 2 ). This association remained strong in the multiple logistic regression model after adjustment for sex, BMI, smoking, diabetes mellitus, HTN, and hypercholesterolemia (Table 2). In the multiple logistic regression model, age, family history of CVD, alcohol consumption, serum creatinine level, and GFR were tested but appeared not to act as independent confounding variables in the current analysis.

All 700 crystal-proven gout patients were enrolled to analyze whether gout disease severity factors were associated with CVD. The clinical characteristics, CV risk factors, and gout disease severity factors of crystal-proven gout patients with and without CVD are shown in Table 3 . The univariate logistic regression analyses revealed that these were univariate risk factors for CVD: gout disease duration $\geq 2$ years, tophaceous gout, oligo- or polyarthritis, $\mathrm{SU}$ acid $>0.55$ $\mathrm{mmol} / \mathrm{l}$, and joint damage (Table 3).

After adding the $\mathrm{CV}$ risk factors in the multivariate logistic regression model, we found that disease duration $\geq 2$ years, oligo- or polyarthritis, $\mathrm{SU}$ acid $(>0.55 \mathrm{mmol} / \mathrm{l})$, and joint damage were still independently associated $(\mathrm{p}<0.05)$ with prevalent CVD after control for age, smoking, diabetes mellitus, and hypercholesterolemia (Table 4). HTN was detected as a univariate risk factor $(\mathrm{p}<0.05)$ in the univariate logistic regression analysis. However, in the full-model multiple logistic regression analysis with backward selection, HTN was not independently associated with prevalent CVD.

\section{DISCUSSION}

According to the results of our study, crystal-proven gout was strongly associated with prevalent CVD compared to non-gout arthritis controls, and characteristic gout severity of crystal-proven gout was positively associated with the presence of CVD.

Table 1. Characteristics and the prevalence of CVD in patients with crystal-proven gout and non-gout arthritis controls. Values are $\mathrm{n}(\%)$ or mean $\pm \mathrm{SD}$ unless otherwise specified.

\begin{tabular}{|c|c|c|c|c|}
\hline Characteristics & Gout, $\mathrm{n}=700$ & Non-gout, $n=276$ & $\mathrm{p}^{\mathrm{a}}$ & OR $(95 \% \mathrm{CI})$ \\
\hline Age, yrs & $62.0 \pm 13.4$ & $58.4 \pm 14.8$ & $<0.001$ & $1.02(1.01-1.03)$ \\
\hline Male sex & $573(81.9)$ & $140(50.7)$ & $<0.001$ & $4.38(3.23-5.94)$ \\
\hline Family history of CVD & $166(23.7)$ & $47(17.0)$ & 0.023 & $1.52(1.07-2.17)$ \\
\hline \multicolumn{5}{|l|}{ Smoking } \\
\hline No & $370(52.9)$ & $149(54.0)$ & $<0.001$ & \\
\hline Alcohol consumption $>21$ units/wk & $190(27.2)$ & $51(18.5)$ & 0.004 & $1.65(1.17-2.33)$ \\
\hline Creatinine level, $\mu \mathrm{mol} / 1$ & $102.5 \pm 40.6^{\mathrm{c}}$ & $77.2 \pm 34.5^{\mathrm{d}}$ & $<0.001$ & $1.04(1.03-1.05)$ \\
\hline $\mathrm{GFR}, \mathrm{ml} / \mathrm{min} / 1.73 \mathrm{~m}^{2}$ & $64.3 \pm 21.5^{\mathrm{e}}$ & $78.32 \pm 16.3^{\mathrm{f}}$ & $<0.001$ & $0.96(0.95-0.97)$ \\
\hline Diabetes mellitus & $123(17.6)$ & $33(12.0)$ & 0.031 & $1.57(1.04-2.37)$ \\
\hline Hypertension & $431(61.6)$ & $92(33.3)$ & $<0.001$ & $3.20(2.39-4.30)$ \\
\hline Hypercholesterolemia & $203(29.0)$ & $44(15.9)$ & $<0.001$ & $2.15(1.50-3.09)$ \\
\hline Heart failure & $98(14.0)$ & $8(2.9)$ & $<0.001$ & $5.45(2.62-11.37)$ \\
\hline Transient ischemic attack & $47(6.7)$ & $10(3.6)$ & 0.064 & $1.92(0.95-3.85)$ \\
\hline Cerebrovascular accident & $47(6.7)$ & $7(2.5)$ & 0.010 & $2.77(1.24-6.20)$ \\
\hline Peripheral vascular disease & $54(7.7)$ & $6(2.2)$ & 0.001 & $3.76(1.60-8.85)$ \\
\hline Heart valve abnormality & $51(7.3)$ & $8(2.9)$ & 0.010 & $2.63(1.23-5.62)$ \\
\hline Arrhythmia & $117(16.7)$ & $21(7.6)$ & $<0.001$ & $2.44(1.50-3.97)$ \\
\hline
\end{tabular}

${ }^{a}$ Based on the differences in both groups after univariate logistic regression analysis. ${ }^{\mathrm{b}}$ Angina pectoris, myocardial infarction, heart failure, transient ischemic attack, cerebrovascular accident, peripheral vascular disease, heart valve abnormality, or arrhythmia. ${ }^{\mathrm{c}} \mathrm{n}=684 .{ }^{\mathrm{d}} \mathrm{n}=258 .{ }^{\mathrm{e}} \mathrm{n}=452 .{ }^{\mathrm{f}} \mathrm{n}=132 . \mathrm{CVD}$ : cardiovascular disease; BMI: body mass index; GFR: glomerular filtration rate. 
Table 2. OR (95\% CI) for crystal-proven gout associated with CVD using controls as a reference.

\begin{tabular}{lcc}
\hline $\mathrm{n}=976^{\dagger}$ & OR $(95 \% \mathrm{CI})$ & $\mathrm{p}$ \\
\hline $\begin{array}{l}\text { Model 1 } \\
\text { Non-gout controls }\end{array}$ & $1.00(\mathrm{ref})$ & \\
$\begin{array}{l}\text { Crystal-proven gout } \\
\text { Model 2 }\end{array}$ & $2.85(2.09-3.91)$ & $<0.001$ \\
$\quad \begin{array}{l}\text { Non-gout controls } \\
\text { Crystal-proven gout }\end{array}$ & $1.00(\mathrm{ref})$ & \\
$\begin{array}{l}\text { Model 3 } \\
\text { Non-gout controls } \\
\text { Crystal-proven gout }\end{array}$ & $3.39(2.37-4.84)$ & $<0.001$ \\
\hline
\end{tabular}

$\dagger$ Crystal-proven gout $(\mathrm{n}=700)$; non-gout controls $(\mathrm{n}=276) .{ }^{\text {a }}$ Model 1 : crude associations. ${ }^{\mathrm{b}}$ Model 2: corrected for age, BMI, and smoking. ${ }^{\mathrm{c}}$ Model 3: corrected for age, BMI, smoking, diabetes mellitus, hypertension, and hypercholesterolemia. CVD: cardiovascular disease; BMI: body mass index.

In agreement with previous studies, we found a high prevalence of CVD in patients with gout ${ }^{9}, 11$. In our study, we found an even higher prevalence (47\%; 95\% CI 44-51\%) of CVD in gout patients, compared to the CVD prevalence in patients with gout in other studies (35\% and 23\%, respectively $)^{9,11}$. In addition, 8 different CVD were examined and all CVD in our study, with the exception of transient ischemic attack, had a statistically significant higher prevalence in patients with crystal-proven gout compared to controls. Our data showed a higher prevalence of myocardial infarction $(15 \%)$, heart failure (14\%) and stroke $(6.7 \%)$ in patients with gout, compared to earlier reports that showed lower preva- lence of myocardial infarction (14\%), heart failure (11\%), and stroke $(10 \%)$ in patients with gout ${ }^{10,11}$.

Moreover, in our study we found a stronger relationship between gout and CVD after confounder correction was attained (adjusted OR 3.39, 95\% CI 2.37-4.84; Table 2, model 2) compared to the literature (age-adjusted OR 2.70) ${ }^{11}$. A likely explanation is that gout in our study was always based on MSU crystal identification, while other studies accepted clinical criteria for the diagnosis of gout ${ }^{2,5-12}$. Misclassification resulting from using the clinical diagnosis of gout could lead to underestimation of the real effect between gout and CVD.

The association between crystal-proven gout and prevalent CVD in our study is higher (adjusted OR 3.39, 95\% CI 2.37-4.84; Table 2, model 2) in comparison with the prevalence of CVD in patients with other inflammatory disorders such as rheumatoid arthritis (RA; adjusted OR 2.70, 95\% CI 1.24-5.86) and ankylosing spondylitis (AS; adjusted OR $1.41,95 \%$ CI $1.29-1.54$ ) after adjustment for conventional CV risk factors, as described in previous literature ${ }^{19,20}$.

In our study, we hypothesized that gout disease severity factors [duration $\geq 2$ years, attack frequency $\geq 4$ attacks/yr, tophaceous gout, oligo- or polyarthritis, high SU acid $(>0.55$ $\mathrm{mmol} / \mathrm{l}$ ), and presence of gout-related joint damage] or features of chronic gout were associated with CVD in gout. Most severity factors, with the exception of tophaceous gout, were associated with having CVD, supporting the hypothesis of a relationship between gout and CVD. It should be noted these severity factors are collected during the first visit at our

Table 3. Characteristics and gout disease severity factors of crystal-proven gout patients $(\mathrm{n}=700)$ with and without prevalent CVD. Values are $\mathrm{n}(\%)$ or mean \pm SD unless otherwise specified.

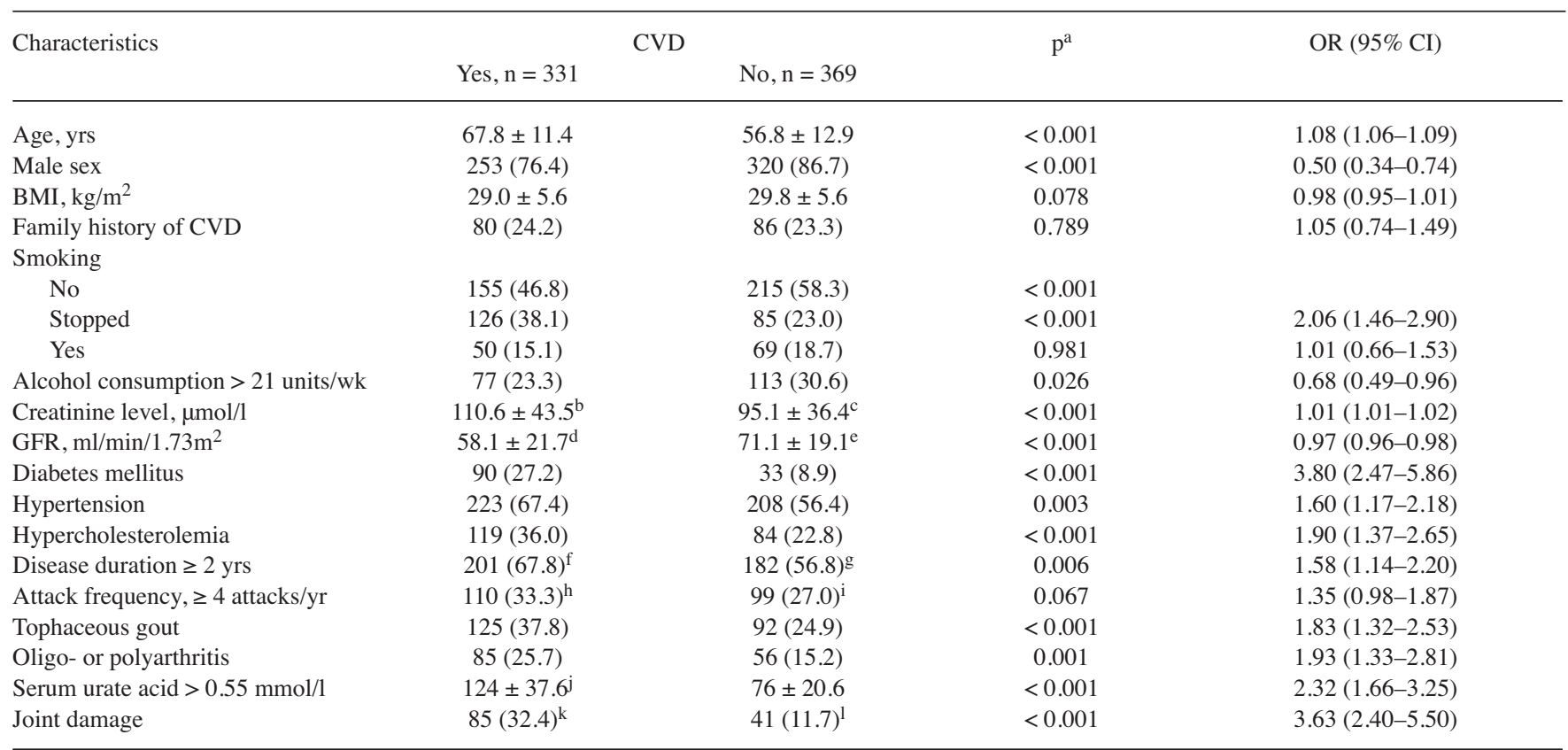

${ }^{a}$ Based on the differences in both groups after univariate logistic regression analysis. ${ }^{b} \mathrm{n}=324 .{ }^{\mathrm{c}} \mathrm{n}=360 .{ }^{\mathrm{d}} \mathrm{n}=239 .{ }^{\mathrm{e}} \mathrm{n}=213 .{ }^{\mathrm{f}} \mathrm{n}=297 .{ }^{\mathrm{g}} \mathrm{n}=320 .{ }^{\mathrm{h}} \mathrm{n}=330$. ${ }^{\mathrm{i}} \mathrm{n}=367 .{ }^{\mathrm{j}} \mathrm{n}=330 .{ }^{\mathrm{k}} \mathrm{n}=262 .{ }^{\mathrm{l}} \mathrm{n}=351$. CVD: cardiovascular disease; BMI: body mass index; GFR: glomerular filtration rate. 
Table 4. Regression coefficients and OR (95\% CI) for risk factors associated with CVD in patients with crystal-proven gout. $\mathrm{N}=700$; significance level of model, $\mathrm{p}<0.001$.

\begin{tabular}{|c|c|c|c|}
\hline Factor & $\beta$ & $\mathrm{p}$ & OR $(95 \% \mathrm{CI})$ \\
\hline Age, yrs & 0.055 & $<0.001$ & $1.06(1.04-1.08)$ \\
\hline Stopped smoking ${ }^{1}$ & 0.700 & 0.002 & $2.01(1.29-3.14)$ \\
\hline Active smoking ${ }^{2}$ & 0.755 & 0.009 & $2.13(1.21-3.74)$ \\
\hline Diabetes mellitus $^{3}$ & 0.834 & 0.003 & $2.30(1.35-3.92)$ \\
\hline Hypercholesterolemia $^{4}$ & 0.533 & 0.017 & $1.70(1.10-2.63)$ \\
\hline Disease duration $\geq 2 \mathrm{yrs}^{5}$ & 0.698 & 0.001 & $2.01(1.31-3.09)$ \\
\hline Oligo- or polyarthritis ${ }^{6}$ & 0.505 & 0.047 & $1.66(1.03-2.66)$ \\
\hline Serum urate acid $>0.55 \mathrm{mmol} / \mathrm{1}^{7}$ & 0.547 & 0.011 & $1.73(1.12-2.68)$ \\
\hline Joint damage $^{8}$ & 0.814 & 0.001 & $2.26(1.38-3.69)$ \\
\hline
\end{tabular}

\footnotetext{
${ }^{1}$ Stopped smoking $=1$; never smoked $=0 .{ }^{2}$ Active smoking $=1$; never smoked $=0 .{ }^{3}$ Diabetes mellitus present $=1$; diabetes mellitus not present $=0$. ${ }^{4}$ Hypercholesterolemia present $=1$; hypercholesterolemia not present $=0$. ${ }^{5}$ Disease duration $\geq 2$ years $=1$; disease duration $<2$ years $=0 .{ }^{6}$ Oligo- or polyarthritis $=1$; monoarthritis $=0 .{ }^{7}$ Serum urate acid $>0.55 \mathrm{mmol} / 1=1$; serum urate acid $\leq 0.55 \mathrm{mmol} / \mathrm{l}=0 .{ }^{8}$ Joint damage present $=1$; joint damage not present $=0$. CVD: cardiovascular disease .
}

outpatient clinic and that severity factors relating to the magnitude of the deposits are most likely associated inflammation. This may imply that CVD, in well-treated patients with gout, is preventable.

A strength of our study is that the diagnosis of gout was always based on MSU-crystal identification. Another strength is the prospective design, with data collection at the inclusion visit. In addition, CV risk factors and CVD were double-checked, with patients being asked for the presence of $\mathrm{CV}$ risk factors and CVD at the inclusion visit. Important misclassification bias concerning the registration was minimized for that reason.

The limitation of our study is the cross-sectional study design. Because gout and CVD were simultaneously assessed, it was unknown whether gout or CVD was first present in patients. Although we found a strong association between gout with gout disease severity and prevalent CVD, our observation does not prove causality if taken in isolation. A second limitation was that we did not collect data at inclusion on whether the patients used urate-lowering prophylaxis. Therefore, the existence of oligo- or polyarthritis may be underestimated. A third limitation is that we did not collect data on treatment duration because this was impractical. The patients are often already treated by general practitioners for unknown periods of time. They often stopped therapy and started again for uncertain periods. Therefore, our study could not give an answer on whether CV risk in patients with gout may improve after proper treatment. A fourth limitation is it cannot be excluded that the control group also have a certain $\mathrm{CV}$ risk, which can influence the risk estimations for the gout group.

Cogswell, et al mentions Hill's 8 criteria to assess causality in an observed association: strength, consistency, specificity, temporality, biologic gradient, plausibility, coherence, and analogy ${ }^{21}$. These all strongly support a causal relationship between the presence of arthritis, such as in gout and CV risk: the consistency of our observation with previous observational studies in patients with a clinical diagnosis of gout (verification without using MSU crystal identification), the strength of the association with a clear dose-response relationship as reflected by the association between gout severity and CV risk in our study, its coherence and analogy with the observed association between $\mathrm{CV}$ risk, and the presence of other types of joint inflammation such as RA and $\mathrm{AS}^{5,6,7,9,10,11,19,20}$. The causal role of gout in CV risk has been further demonstrated by experimental evidence in animals that an artificial increase in SU acid by inhibition of uricase or diet induces atherosclerosis and the development of $\mathrm{HTN}^{22,23,24}$. Interestingly, in our database HTN was associated with CVD but this association was lost when corrected for the presence of gout, suggesting an overlap in the pathophysiological role of HTN and gout in development of atherosclerosis. This is supported by experimental evidence that hyperuricemia is a cause of $\mathrm{HTN}^{23}$. A wealth of biomedical research is currently available indicating the plausibility for a causal role for inflammation in the pathogenesis of atherosclerosis $^{25}$. In addition, urate may induce direct endothelial toxicity $^{26}$. However, experimental evidence in humans is scarce and not unequivocal. Although reduction of uric acid with allopurinol improves vascular function and reduces blood pressure, intraarterial infusion of uric acid does not affect endothelial function in healthy volunteers. A published Mendelian randomization study found a causal relation between uric acid and the occurrence of gout, but could not confirm this for CVD $^{27,28,29,30,31}$. Several explanations can be put forward for these conflicting results in humans in vivo. Allopurinol not only reduces SU acid but also reduces formation of reactive oxygen species by inhibiting xanthine oxidase, providing an alternative explanation for its observed benefit on vascular vasodilator function ${ }^{32}$. The vasculotoxic actions of uric acid may depend on a metabolic and proinflammatory background as offered by the metabolic syndrome, which may in part be caused by prolonged hyperuricemia ${ }^{33}$. In addition, in the Mendelian randomization study, pleiotropic effects of studied genes may have blurred a causal role of gout in the development of atherosclerosis. Thus, in the context of previous observational and preclinical experimental studies ${ }^{2,5-12}$, the claim of a causal role of gout in development of atherosclerosis is strong. Our study adds to this claim by providing evidence for a biological gradient (severity of gout associated with CV disease) and an increased strength of the association with a stricter diagnosis of gout by demonstration of intraarticular MSU crystals. Finally, experimental and trial evidence in humans is needed that demonstrates clinically relevant $\mathrm{CV}$ benefit by reducing SU and/or prevention of gout-associated inflammation.

Our study showed that crystal-proven gout was strongly associated with prevalence of CVD. Within gout patients,

Personal non-commercial use only. The Journal of Rheumatology Copyright @ 2018 . All rights reserved. 
univariate risk factors for CVD were gout disease duration $\geq 2$ years, oligo- or polyarthritis, high $\mathrm{SU}$ acid $(>0.55$ $\mathrm{mmol} / \mathrm{l})$, and joint damage. Moreover, these were independently associated with prevalent CVD: disease duration $\geq 2$ years, oligo- or polyarthritis, SU acid (>0.55 mmol/l) and joint damage. Application of Hill's criteria as found in Cogswell, et $a l^{21}$ indicates that the claim of a causal role of gout in the development of CVD is strong. Experimental evidence in humans is needed that demonstrates clinically relevant $\mathrm{CV}$ benefit by reducing SU and/or prevention of gout-associated inflammation.

\section{REFERENCES}

1. Annemans L, Spaepen E, Gaskin M, Bonnemaire M, Malier V, Gilbert T, et al. Gout in the UK and Germany: prevalence, comorbidities and management in general practice 2000-2005. Ann Rheum Dis 2008;67:960-6.

2. Singh JA. When gout goes to the heart: does gout equal a cardiovascular disease risk factor? Ann Rheum Dis 2015;74:631-4.

3. Khanna D, FitzGerald JD, Khanna PP, Bae S, Singh M, Neogi T, et al. 2012 American College of Rheumatology guidelines for management of gout part I: systematic non-pharmacologic and pharmacologic therapeutic approaches to hyperuricemia. Arthritis Care Res 2012;64:1431-46.

4. Bhansing KJ, van Bon L, Janssen M, Radstake TR. Gout: a clinical syndrome illustrated and discussed. Neth J Med 2010;68:352-9.

5. Kuo CF, See LC, Luo SF, KO YS, Lin YS, Hwang JS, et al. Gout: an independent risk factor for all-cause and cardiovascular mortality. Rheumatology 2010;49:141-6.

6. Sattui SE, Singh JA, Gaffo AL. Comorbidities in patients with crystal diseases and hyperuricemia. Rheum Dis Clin North Am 2014;40:251-78.

7. Krishnan E, Svendsen K, Neaton JD, Grandits G, Kuller LH. Long-term cardiovascular mortality among middle-aged men with gout. Arch Intern Med 2008;168:1104-10.

8. Dutch Society for Rheumatology. [Guidelines for Gout 2013]. [Article in Dutch] [Internet. Accessed February 5, 2018.] Available from: www.nvr.nl/wp-content/uploads/2014/11/ NVR-Reumatische-ziekten-richtlijn-jicht-2013.pdf

9. Janssens HJ, van de Lisdonk EH, Bor H, van den Hoogen HJ, Janssen M. Gout, just a nasty event or a cardiovascular signal? A study from primary care. Fam Pract 2003;20:413-6.

10. Zhu Y, Pandya BJ, Choi HK. Comorbidities of gout and hyperuricemia in the US general population: NHANES 2007-2008. Am J Med 2012;125:679-87.

11. Winnard D, Wright C, Jackson G, Gow P, Kerr A, McLachlan A, et al. Gout, diabetes and cardiovascular disease in the Aotearoa New Zealand adult population: co-prevalence and implications for clinical practice. N Z Med J 2013;126:53-64.

12. Kiltz U, Alten R, Fleck M, Kruger K, Manger B, Muller-Ladner U, et al. [Guideline report on the methodology of the S2e guideline: Gouty arthritis.] [Article in German] [Internet. Accessed February 5, 2018.] Available from: www.awmf.org/uploads/tx_szleitlinien/ 060-005m_S2e_Gichtarthritis_2016-06.pdf 2016

13. Zhang W, Doherty M, Pascual E, Bardin T, Barshova V, Conaghan $\mathrm{P}$, et al; EULAR Standing Committee for International Clinical Studies Including Therapeutics. EULAR evidence based recommendations for gout. Part I: Diagnosis. Report of a task force of the Standing Committee for International Clinical Studies Including Therapeutics (ESCISIT). Ann Rheum Dis 2006; 65:1301-11.
14. Liu WC, Hung CC, Chen SC, Yeh SM, Lin MY, Chiu YW, et al Association of hyperuricemia with renal outcomes, cardiovascular disease, and mortality. Clin J Am Soc Nephrol 2012;7:541-8.

15. Janssens HJ, Fransen J, van de Lisdonk EH, van Riel PL, van Weel C, Janssen M. A diagnostic rule for acute gouty arthritis in primary care without joint fluid analysis. Arch Int Med 2010;170:1120-6.

16. Kienhorst LB, Janssens HJ, Fransen J, Janssen M. The validation of a diagnostic rule for gout without joint fluid analysis: a prospective study. Rheumatology 2015;54:609-14.

17. Perez-Ruiz F, Dalbeth N, Urresola A, de Miguel E, Schlesinger N. Imaging of gout: findings and utility. Arthritis Res Ther 2009;11:232.

18. Dalbeth N, Clark B, McQueen F, Doyle A, Taylor W. Validation of a radiographic damage index in chronic gout. Arthritis Rheum 2007;57:1067-73

19. van Halm VP, Peters MJ, Voskuyl AE, Boers M, Lems WF, Visser $\mathrm{M}$, et al. Rheumatoid arthritis versus diabetes as a risk factor for cardiovascular disease: a cross-sectional study, the CARRE Investigation. Ann Rheum Dis 2009;68:1395-400.

20. Ungprasert P, Srivali N, Kittanamongkolchai W. Risk of coronary artery disease in patients with ankylosing spondylitis: a systematic review and meta-analysis. Ann Transl Med 2015;3:51.

21. Cogswell ME, Mugavero K, Bowman BA, Frieden TR. Dietary sodium and cardiovascular disease risk - measurement matters. N Engl J Med 2016;375:580-6.

22. Liu Z, Chen T, Niu H, Ren W, Li X, Cui L, et al. The Establishment and characteristics of rat model of atherosclerosis induced by hyperuricemia. Stem Cells Int 2016;2016:1365257.

23. Mazzali M, Hughes J, Kim YG, Jefferson JA, Kang DH, Gordon $\mathrm{KL}$, et al. Elevated uric acid increases blood pressure in the rat by a novel crystal-independent mechanism. Hypertension 2001; 38:1101-6.

24. Khosla UM, Zharikov S, Finch JL, Nakagawa T, Roncal C, Mu W, et al. Hyperuricemia induces endothelial dysfunction. Kidney Int 2005;67:1739-42

25. Hansson GK, Libby P, Tabas I. Inflammation and plaque vulnerability. J Intern Med 2015;278:483-93.

26. Hong Q, Qi K, Feng Z, Cui S, Wang L, Fu B, et al. Hyperuricemia induces endothelial dysfunction via mitochondrial $\mathrm{Na}+\mathrm{Ca} 2+$ exchanger-mediated mitochondrial calcium overload. Cell Calcium 2012;51:402-10.

27. Yelken B, Caliskan Y, Gorgulu N, Altun I, Yilmaz A, Yazici H, et al. Reduction of uric acid levels with allopurinol treatment improves endothelial function in patients with chronic kidney disease. Clin Nephrol 2012;77:275-82.

28. Soletsky B, Feig DI. Uric acid reduction rectifies prehypertension in obese adolescents. Hypertension 2012;60:1148-56.

29. Agarwal V, Hans N, Messerli FH. Effect of allopurinol on blood pressure: a systematic review and meta-analysis. J Clin Hypertens 2013;15:435-42.

30. Waring WS, Adwani SH, Breukels O, Webb DJ, Maxwell SR. Hyperuricaemia does not impair cardiovascular function in healthy adults. Heart 2004;90:155-9.

31. Keenan T, Zhao W, Rasheed A, Ho WK, Malik R, Felix JF, et al. Causal assessment of serum urate levels in cardiometabolic diseases through a Mendelian randomization study. J Am Coll Cardiol 2016;67:407-16.

32. Rajendra NS, Ireland S, George J, Belch JJ, Lang CC, Struthers AD. Mechanistic insights into the therapeutic use of high-dose allopurinol in angina pectoris. J Am Coll Cardiol 2011;58:820-8.

33. Nakagawa T, Hu H, Zharikov S, Tuttle KR, Short RA, Glushakova $\mathrm{O}$, et al. A causal role for uric acid in fructose-induced metabolic syndrome. Am J Physiol Renal Physiol 2006;290:F625-31. 